Current Issues of Education and Science, 2021

DOI: $\underline{\text { https://doi.org/10.26697/9786177089147.2021.12 }}$

\title{
Study of the Phenopatotypes of Clinical Isolates of Dominant Species of Staphylococcus, Isolated from Patients with Atopic Dermatitis
}

\author{
Svetlana K. DZHORAIEVA ${ }^{1}$ (iD), Yuliia V. SHCHERBAKOVA ${ }^{1}$ iD, \\ Irina V. ZUBAN ${ }^{1}$ iD \\ ${ }^{1}$ Institute of Dermatology and Venereology of the National Academy \\ of Medical Sciences of Ukraine, Ukraine
}

Corresponding Author Details

Svetlana K. DZHORAIEVA, dzhoraevasvetlana@gmail.com

Received: 23.09.2021; Accepted: 21.10.2021; Published: 17.12.2021

\section{Brief Summary}

Background: Atopic dermatitis $(A D)$ occupy the main place among dermatoses, where the allergic component is leading in the onset and development of the disease. The most common complication of allergic dermatitis is the attachment of a secondary pyococcus infection, which is associated with a decrease in the antimicrobial resistance of skin surface.

The aim of the study: To determine phenotypic features of staphylococci extracted from patients with allergic dermatitis to assess their pathogenic potential.

Methods: The object of the study was staphylococcus isolates removed from affected and intact skin sections of patients with $A D$, as well as from representative skin sections of healthy individuals undergoing inpatient treatment at the Department of Dermatology of Institute of Dermatology and Venereology.

Results: It's established that the complex of phenotypic traits of the removed staphylococcus cultures indicates the presence in the pathogen of factors related to the resistance of the host protection mechanisms and determines the intensity of the alterative action of the infectant in relation to 
the host organism, the phenotypic manifestation of the studied factors was higher in the staphylococcus isolates removed from the affected skin areas of patients with allergic dermatitis.

Conclusions: The level and frequency of phenotypic expression of pathogenicity factors are more pronounced in microorganisms obtained from patients from affected and intact areas compared to controls, which confirms their pathogenetic role in the burden of the disease, which in turn can be used as an auxiliary differential diagnosis criterion.

\section{Keywords:}

clinical strains of staphylococci, atopic dermatitis, infectious complications, pathogenicity factors.

\section{Background:}

Atopic dermatitis occupies the main place among dermatoses, where the allergic component is leading in the onset and development of the disease (Belkaid \& Segre, 2014; Kantor et al., 2016). The most common complication of allergic dermatitis is the attachment of a secondary pyococcus infection, which is associated with a decrease in the antimicrobial resistance of the skin surface (Grice et al., 2008; Nada et al., 2012; Rojo et al., 2014). In this case, the leading role is played by Staphylococcus aureus, which is seeded in $80-95 \%$ of patients diagnosed with AD. The clinical significance of the pathogen is determined by its ability to actively survive in the biotope, aided by a wide range of pathogenicity factors of this microorganism. Therapy of infectious lesions is complicated by the increasing resistance of the main pathogens of pyoderma - Staphylococcus aureus and Staphylococcus epidermidis - to widely used antibiotics (Kutasevych et al., 2019; Oh et al., 2014).

The aim of the study. To determine the phenotypic features of staphylococci extracted from patients with allergic dermatitis to assess their pathogenic potential.

\section{Methods:}

The object of the study was staphylococcus isolates removed from affected and intact skin sections of patients with $\mathrm{AE}$, as well as from representative skin sections of healthy individuals undergoing inpatient treatment at the Department of Dermatology of "Institute of Dermatology and Venereology of NAMS of Ukraine". Biochemical identification and biological properties of staphylococci were determined using methods of classical bacteriology. 


\section{Results:}

According to the obtained results of microbiological studies, the presence of a direct (positive) correlation between the severity of the course of dermatosis and the level of microbial contamination of both the lesions and the intact skin areas of patients is proved. The highest rates of microbial colonization density of affected skin areas were found in groups of patients with severe $\mathrm{AD}\left(7.0 \pm 0.2 \mathrm{lg} \mathrm{CFU} / \mathrm{cm}^{2}\right)$. In patients with $\mathrm{AD}$, a direct relationship was established between the severity of dermatosis and the frequency of S. aureus isolation from lesions and intact areas of the skin: $46.7 \%$ and $38.5 \%$ for mild cases $59.4 \%$ and $45.2 \%$ for moderate cases, $81.8 \%$ and $70.4 \%$ for severe cases. However, the frequency of S. epidermidis removal from the same groups of patients with AD had an inverse relationship: $20.0 \%$ and $30.8 \%, 15.6 \%$ and $19.4 \%$, and $10.9 \%$ and $16.7 \%$, respectively. Analysis of the enzymatic activity of isolates of dominant species of Staphylococcus removed from the skin lesions of patients with allergodermatoses, revealed that all strains of S. aureus displayed coagulase, lecithinase and hemolytic activity. The latter was also found in all S. haemolyticus strains and in $55.4 \%$ of S. epidermidis strains. The presence of lipolytic activity was determined in $74.7 \%$ of S. aureus strains, $82.7 \%$ of S. haemolyticus strains and $76.8 \%$ of S. epidermidis strains. Proteolytic activity, with corresponding manifestations of milk fermentation and gelatin dilution, was inherent in $95.3 \%$ and $82.9 \%$ of S. aureus strains, $49.3 \%$ and $24.0 \%$ of S. haemolyticus strains, as well as $73.2 \%$ and $19.6 \%$ of S. epidermidis strains, respectively.

According to the results of the study of adhesion-colonization potential of strains of staphylococci of different origin, it was found that the highest levels of the average adhesion index (AAI) and microbial adhesion index (MAI) were discovered in the group of strains removed from damaged skin areas of patients with allergic dermatoses $(p \leq 0.05)$. At the same time, their values reached: AAI $(3.5 \pm 0.2)$ of bacteria/erythrocyte and MAI (4.2 \pm 0.3$)$ of bacteria/erythrocyte, which is almost 1.5 times higher than in the group of isolated strains from intact skin areas (respectively: $(2.4 \pm 0.2)$ and $(2.8 \pm 0.2)$ bacteria/erythrocyte) and 3 times higher than the values of similar indicators/indices of the control group of strains isolated

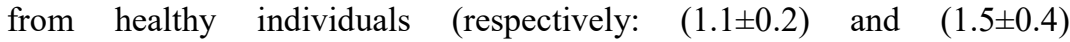
bacteria/erythrocyte). In the study of anti-lysozyme activity (ALA) in strains of staphylococci isolated from patients with allergodermatoses and from healthy individuals, it was found that $87.7 \%$ of the strains isolated from the affected skin areas of patients with a severe disease course, while in isolates from intact skin areas of theme-based patients and from healthy individuals the presence of ALA was found at the level of $48.8 \%$ and 
$17.6 \%$, respectively $(\mathrm{p} \leq 0.05)$. In addition, it was found that the strains of Staphylococcus removed from the areas of the affected skin had the highest and absolute rates of ALA, which reached the strains of S. aureus $(3.0 \pm 0.2) \mu \mathrm{g} / \mathrm{ml}, \quad$ S. haemolyticus $\quad(2.2 \pm 0.1) \mu \mathrm{g} / \mathrm{ml}$ and $\quad$ S. epidermidis $(1.3 \pm 0.1) \mu \mathrm{g} / \mathrm{ml}$. According to the research of anti-interferon activity (AIA) of groups of Staphylococcus strains of different origin and species, it was found that $66.7 \%$ of Staphylococcus strains isolated from the affected skin areas of patients with allergodermatoses, $58.1 \%$ was isolated from intact skin areas and only $35.3 \%$ of them was isolated from the skin of healthy persons $(\mathrm{p} \leq 0.05)$. The level of expression of this trait in $\mathrm{S}$. aureus strains removed from any (both affected and intact) areas of patients' skin was high and ranged from 2.0 to $2.3 \mathrm{IU}$.

In determining the sensitivity of $\mathrm{S}$. aureus strains removed from patients with allergodermatoses to anti-bacterial drugs of different chemical groups ( $\mathrm{ABD}$ strains, the highest $(\mathrm{p} \leq 0.05)$ sensitivity values were established for fusidic acid $(90.8 \%$ and $92.3 \%$, respectively) and oxazolidinones (92.1\% and $93.7 \%$, respectively), suggesting the latter for use as $\mathrm{ABD}$ at the initial stage of urgent treatment of severe forms of infectious-inflammatory complications to obtain the result of microbiological examination with determination of the actual antibiotic sensitivity of pathogens removed from locus morbi of patients with atopic dermatitis.

\section{Conclusions:}

Thus, the complex of phenotypic features of the removed staphylococcus cultures indicates the presence in the pathogen of factors associated with the resistance of host protection mechanisms, on the one hand, and high pathogenic potential, on the other, which promotes active colonization of both affected and intact areas of the skin, which provides the conditions for long-term persistence and determines the intensity of the alterative action of the infectious agent against the host organism.

The severity of anti-lysozyme and anti-interferon activities in strains isolated from affected skin areas was significantly higher than in cultures isolated from intact skin areas both qualitatively and quantitatively. The definition of high and moderate values of these signs for $\mathrm{S}$. aureus strains in lesions compared with healthy skin suggests a certain complicating role of this factor for the development of AD.

The level and frequency of phenotypic expression of pathogenicity factors are more pronounced in microorganisms obtained from patients from affected and intact areas compared to controls, which confirms their 
pathogenetic role in the burden of the disease, which in turn can be used as an auxiliary differential diagnosis criterion.

\section{Conflicts of interests:}

The authors declare that there is no conflict of interests.

\section{Source of support:}

This research did not receive any outside funding or support.

\section{References}

Belkaid, Y., \& Segre, J. A. (2014). Dialogue between skin microbiota and immunity. $\quad$ Science, $346(6212), \quad 954-959$. https://doi.org/10.1126/science.1260144

Grice, E. A., Kong, H. H., Renaud, G., Young, A. C., Bouffard, G. G., Blakesley, R. W., Wolfsberg, T. G., Turner, M. L., \& Segre, J. A. (2008). A diversity profile of the human skin microbiota. Genome Research, 18(7),

1043-1050. https://doi.org/10.1101/gr.075549.107

Kantor, R., Thyssen, J. P., Paller, A. S., \& Silverberg, J. I. (2016). Atopic dermatitis, atopic eczema, or eczema? A systematic review, metaanalysis, and recommendation for uniform use of 'atopic dermatitis'. Allergy, 71(10), 1480-1485. https://doi.org/10.1111/all.12982

Kutasevych, Ya., Dzhoraeva, S., Shcherbakova, Yu., Bondarenko, G., \& Sobol, N. (2019). Studying the pathogenic properties of the skin's autoflora in patients diagnosed with atopic dermatitis. Georgian Medical News, 7-8(292-293), 113-117. https://pubmed.ncbi.nlm.nih.gov/31560675/

Nada, H. A., Gomaa, N. I., Elakhras, A., Wasfy, R., \& Baker, R. A. (2012). Skin colonization by superantigen-producing Staphylococcus aureus in Egyptian patients with atopic dermatitis and its relation to disease severity and serum interleukin-4 level. International Journal of Infectious Diseases, 16(1), e29-e33. https://doi.org/10.1016/j.ijid.2011.09.014

Oh, J., Byrd, A. L. , Deming, C., Conlan, S., Kong, H. H., \& Segre, J. A. (2014). Biogeography and individuality shape function in the human skin metagenome. Nature, 514, 59-64. https://doi.org/10.1038/nature13786

Rojo, A., Aguinaga, A., Monecke, S., Yuste, J. R., Gastaminza, G., \& España, A. (2014). Staphylococcus aureus genomic pattern and atopic dermatitis: may factors other than superantigens be involved? European Journal of Clinical Microbiology \& Infectious 
Diseases, 33(4), 651-658. https://doi.org/10.1007/s10096-0132000-z

\section{Information about the authors:}

Dzhoraieva Svetlana Kariahdyivna - https://orcid.org/0000-0003-24865474; PhD, MD, Doctor of Medicine, Head of Laboratory and Experimental Department, Institute of Dermatology and Venereology of the National Academy of Medical Sciences of Ukraine, Kharkiv, Ukraine.

Research interests: microbiology, dermatology and sexually transmitted infections, diagnostics, biosafety.

Shcherbakova Yuliia Valeriivna - https://orcid.org/0000-0002-36827451; PhD, MD, Doctor of Medicine, Senior Researcher, Scientific Secretary, Institute of Dermatology and Venereology of the National Academy of Medical Sciences of Ukraine, Kharkiv, Ukraine.

Research interests: dermatology and sexually transmitted infections.

Zuban Irina Volodymurivna - https://orcid.org/0000-0003-1323-6509; Graduate Student, Institute of Dermatology and Venereology of the National Academy of Medical Sciences of Ukraine, Kharkiv, Ukraine.

Research interests: dermatology and sexually transmitted infections.

\section{Cite this article as:}

Dzhoraieva, S. K., Shcherbakova, Yu. V., \& Zuban, I. V. (2021). Study of the phenopatotypes of clinical isolates of dominant species of staphylococcus, isolated from patients with atopic dermatitis. In Yu. B. Melnyk, \& L. M. Georgieva (Eds.), Current Issues of Education and Science. 9th International Conference, CIES-2021, Riga, Latvia - Kharkiv, Ukraine, November 10-13, 2021, Conference proceedings (pp. 98-103). KRPOCH. https://doi.org/10.26697/9786177089147.2021.12

\section{Copyright information:}
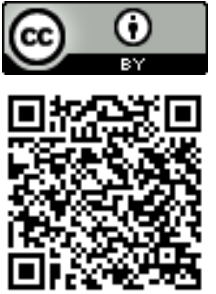

The published paper are licensed under a Creative Commons "Attribution" 4.0 Worldwide

The electronic version of this article is complete. Full or partial reproduction of article is allowed, citing to the source, author(s) and DOI. An electronic copy of the Conference proceedings in open access is available via the $\mathrm{KRPOCH}$ Publishing website https://publisher.culturehealth.org 